\title{
IMAGING
}

\section{Tumor characteristics of urothelial carcinoma on multidetector computerized tomography urography}

Wang LJ, Wong YC, Ng KF, Chuang CK, Lee SY, Wan YL

Department of Medical Imaging and Intervention, Chang Gung Memorial Hospital, College of Medicine, Chang

Gung University, Taoyuan, Taiwan, Republic of China

J Urol. 2010; 183: 2154-60

Purpose: We investigated the relationship between tumor characteristics of urothelial carcinoma and detectability on multidetector computerized tomography urography.

Materials and Methods: We retrospectively reviewed all adult consecutive patients with hematuria who underwent multidetector computerized tomography urography during a 23-month period at our hospital. Patients with a final diagnosis of urothelial carcinoma verified by histological examination of surgical specimens were included in the study. The presence and location of urothelial carcinomas on multidetector computerized tomography urography without knowledge of final diagnosis were recorded. Tumor characteristics (location, size, histological classification and stage) were recorded based mainly on histological findings. The association between tumor characteristics and urothelial carcinoma detectability on multidetector computerized tomography urography was analyzed.

Results: A total of 70 patients who underwent multidetector computerized tomography urography had 87 verified urothelial carcinomas. Of these carcinomas $6(6.9 \%)$ were undetectable by multidetector computerized tomography urography, including 5 ureteral and 1 bladder urothelial carcinoma. Size of detectable and nondetectable tumors on multidetector computerized tomography urography differed significantly $(3.05+/-1.79 \mathrm{vs}$ $0.65+/-0.99 \mathrm{~cm}$, respectively, $\mathrm{p}=0.001)$. Tumor location $(\mathrm{p}=0.009)$, tumor size $1 \mathrm{~cm}$ or larger $(\mathrm{p}=0.003)$ and noncarcinoma in situ tumors $(\mathrm{p}=0.001)$ were significantly associated with multidetector computerized tomography urography detectability. Conversely organ confined disease had no association with multidetector computerized tomography urography detectability. Multivariate analyses showed that noncarcinoma in situ tumor was a significant predictor of multidetector computerized tomography urography detectability $(\mathrm{p}=0.001)$.

Conclusions: Multidetector computerized tomography urography is useful for detecting nearly all urothelial carcinomas in adults with hematuria. Careful assessment by multidetector computerized tomography urography is needed to detect small (less than $1 \mathrm{~cm}$ ) or ureteral urothelial carcinomas. It remains a challenge to detect carcinoma in situ tumors by multidetector computerized tomography urography. Thus, negative results of urothelial carcinomas on multidetector computerized tomography urography do not exclude the presence of carcinoma in situ tumors.

\section{Editorial Comment}

Multidetector CT-urography (MDCT-urography) has been shown to be an effective single comprehensive examination in the evaluation of patients with hematuria or with risk for the development of urothelial malignancies. In this manuscript a total of 201 adults underwent MDCT- urography as imaging investigation of hematuria. Interesting point to consider is number of patients in whom this test was important to determine the etiology of hematuria. Seventy patients (34\%), had urothelial cancer and other $88(43 \%)$ had other urological abnormalities responsible for the hematuria. Specifically in patients with urothelial cancer, $85.7 \%$ presented with gross hematuria and $14.3 \%$ presented with microscopic hematuria. These results emphasize the value of MDCT-urography as a tool for investigation of either gross or microscopic hematuria particularly in older patients. In this series $7 \%$ of tumors were undetectable by MDCT- urography, including 5 ureteral and 1 bladder 
urothelial carcinoma. There are a variety of reasons for false-negative diagnoses of ureteral and bladder cancer during MDCT- urography. Early-enhanced thin-section MDCT of a full bladder with urine can decrease the number of false negatives bladder studies. This "bladder -wall phase ", obtained 60 seconds after contrast injection has superior accuracy for detection of small lesions in comparison with the excretory phase alone (bladder fully distended by opacified urine), as used by the authors. However small flat tumors that do not appear as filling defects and carcinoma in situ tumors, are almost impossible to be detected by MDCT-urography. For this reason, although not a perfect test, cystocopy remains the reference standard procedure in the investigation of hematuria.

Dr. Adilson Prando

Head, Department of Radiology and

Diagnostic Imaging, Vera Cruz Hospital

Campinas, São Paulo, Brazil

E-mail: adilson.prando@gmail.com

doi: 10.1590/S1677-55382010000500019

\section{Prostate cancer managed with active surveillance: role of anatomic MR imaging and MR spectroscopic imaging}

Fradet V, Kurhanewicz J, Cowan JE, Karl A, Coakley FV, Shinohara K, Carroll PR

Department of Urology, Helen Diller Family Comprehensive Cancer Center, University of California, San Francisco, San Francisco, California, USA

Radiology. 2010; 256: 176-83

Purpose: To determine the role that magnetic resonance (MR) imaging and MR spectroscopic imaging findings obtained at the time of diagnosis play in the progression of disease in patients whose prostate cancer is being managed with active surveillance and to compare the role of these findings with the role of transrectal ultrasonography (US) findings.

Materials and Methods: The institutional review board approved this HIPAA-compliant retrospective study, and informed consent was obtained from all patients whose records were to be entered into the research database. All patients who had prostate cancer managed with active surveillance and who had undergone both MR imaging and MR spectroscopic imaging of the prostate and transrectal US at time of diagnosis were identified. Two urologists blinded to the clinical outcome in these patients independently reviewed and dichotomized the MR imaging report and the MR spectroscopic imaging report as normal or suggestive of malignancy. One experienced urologist performed all US examinations that were then dichotomized similarly. Uni- and multivariate (with use of standard clinical variables) Cox models were fitted to assess time to cancer progression, defined as Gleason score upgrading, prostate-specific antigen velocity of more than 0.75 (microg x L(-1))/y, or initiation of treatment more than 6 months after diagnosis.

Results: The final cohort included 114 patients with a median follow-up of 59 months. Patients with a lesion that was suggestive of cancer at MR imaging had a greater risk of the Gleason score being upgraded at subsequent biopsy (hazard ratio, 4.0; 95\% confidence interval: 1.1, 14.9) than did patients without such a lesion. Neither MR spectroscopic imaging nor transrectal US could be used to predict cancer progression.

Conclusion: Abnormal prostate MR imaging results suggestive of cancer may confer an increased risk of Gleason score upgrade at subsequent biopsy. Although expensive, prostate MR imaging may help in counseling potential candidates about active surveillance. 